\title{
El movimiento universitario de Valparaíso y el surgimiento de una nueva izquierda en Chile
}

\author{
Aaron Briceño Arévalo ${ }^{1}$
}

Fecha de recepción: 18 de marzo de 2019

Fecha de aprobación: 6 de mayo de 2019

Fecha de publicación: 30 de julio de 2019

\begin{abstract}
Resumen
El presente artículo propone un análisis de la trayectoria del movimiento estudiantil universitario de Valparaíso en el periodo 2006- 2016, y su relación con la emergencia de una nueva izquierda en Chile. La investigación se construyó bajo una metodología cualitativa, a partir de entrevistas a exdirigentes/as universitarios en el periodo antes señalado. A partir de lo anterior, revisamos la trayectoria de luchas y organización del movimiento universitario, fijando como hito de inicio el 2006, dada la importancia histórica de dicha movilización secundaria. Se problematiza respecto del concepto de "nueva izquierda" y las características que ésta ha adoptado en el nuevo ciclo político chileno, fijando como límite de análisis el año 2016, fecha en que estas izquierdas asumen cargos de representación institucional.
\end{abstract}

Palabras clave: movimiento estudiantil, nuevas izquierdas, posdictadura, Valparaíso

\section{The Valparaiso university movement and the emergence of a new left in Chile}

\begin{abstract}
This article proposes an analysis of the Valparaíso university student movement between 2006 and 2016, and its relationship to the emergence of a new left-wing in Chile. Research is based on qualitative methodology, with data collected through interviews of former university student leaders during this period. Based on the above, this paper examines the movement's history of struggles and its organization, of organization and struggles is examined, dating back to 2006 with the historical relevance of the high school student protests that took place that year. Also, the idea of a "new left" is defined as well the characteristics it has adopted within the new Chilean political cycle. The analysis goes only up to 2016, when these left-wing organizations started assuming positions of institutional representation.
\end{abstract}

Keywords: student movement, new left, post-dictatorship, Valparaíso

Sociólogo, Universidad de Valparaíso, Chile. Contacto: aaron.briceno@gmail.com 


\title{
O movimento universitário de Valparaíso e o surgimento de uma nova esquerda no Chile
}

\begin{abstract}
Resumo
O presente artigo propõe uma análise da trajetória do movimento estudantil universitário de Valparaíso no período 2006- 2016, e sua relação com a emergência de uma nova esquerda no Chile. A pesquisa construiu-se sob uma metodologia qualitativa, a partir de entrevistas a ex-dirigentes universitários no período antes indicado. A partir do anterior, revisamos a trajetória de lutas e organização do movimento universitário, estabelecendo como fato marcante o início de 2006, dada a importância histórica de dita mobilização secundária. Problematiza-se em torno do conceito de "nova esquerda" e as caraterísticas que esta tem adoptado no novo ciclo político chileno, fixando como limite de análise o ano 2016, data em que estas esquerdas assumem cargos de representação institucional.
\end{abstract}

Palavras-chave: movimento estudantil, novas esquerdas, pós-ditadura, Valparaíso

\section{Introducción}

Para comprender el proceso de deslegitimación de las clases dominantes y las perspectivas de cambio que se han instalado en la última década en nuestros territorios, resulta necesario volcarse hacia los movimientos sociales, los que han resquebrajado el silencio impuesto en los primeros años de la transición política, al poner en entredicho las desigualdades del sistema en su conjunto, instalando una crítica profunda al neoliberalismo chileno, lo que en el plano estudiantil se expresó en el cuestionamiento al modelo de mercado y a las políticas educativas de carácter vertical, centralista y extranjerizante desarrolladas desde la década de los ochenta (Reyes, 2014). Diversas investigaciones han dado cuenta del potencial histórico de las movilizaciones estudiantiles de la última década, vinculando los procesos de fortalecimiento de los movimientos con la desvinculación entre la sociedad civil y las instituciones estatales de representación (Garcés, 2012), proceso que posibilitó el despertar de la sociedad y el cuestionamiento del sentido común neoliberal (Ruiz, 2015), pero también la reaparición de las izquierdas en la escena pública tras décadas de exclusión (Gómez Leyton, 2010). 
El renacer del movimiento estudiantil en el nuevo siglo no puede comprenderse de manera aislada, sino relacionado con el contexto continental de reemergencia de los movimientos sociales que cuestionan el orden neoliberal y sus consecuencias sobre la vida y los territorios (Zibechi, 2010), pero que también es consecuencia de un largo proceso de latencia que, poco a poco, le permitió superar las estructuras de contención que operaban sobre el movimiento, muy efectivas en los primeros años de la posdictadura. En este sentido, podemos ver cómo las generaciones estudiantiles del nuevo siglo comienzan un lento resurgir, que se cataliza en los procesos de movilización de las últimas dos décadas (Briceño Arévalo, 2018).

No podríamos referirnos a la actualidad del movimiento estudiantil y de las organizaciones de izquierda que se desarrollaron a su alero sin mencionar las profundas transformaciones al Estado y la sociedad que se impusieron durante la dictadura cívico-militar de Augusto Pinochet (1973-1990). Pues, seguido de la instauración de un Estado policial-autoritario, que reprimió abiertamente a la población y dinamitó los procesos de politización y acumulación de fuerzas en el campo popular, reorganizó el país sobre nuevas bases económicas, políticas y sociales, todas consecuentes con el ideario neoliberal (Goicovic, 2006). El sistema educativo, en su conjunto, se refundó bajo estas nuevas lógicas; de este modo, la reorganización neoliberal operó en distintos planos y niveles, todos conducentes a desmantelar el radio de acción del Estado, promoviendo la inversión privada en desmedro del gasto público, así como también imponiendo políticas de disciplinamiento y competencia entre los/as actores educativos/as y una desorientación epistemológica que ha derivado en la reproducción, sin mayores contrapesos, de los valores y los patrones dominantes en la producción de conocimientos y en la transformación de estos en mercancías transables, formando por tanto sobre la base de las competencias que requiere el mercado (De Sousa Santos, 2008; Giroux, 2011). 
Estos procesos afectaron fuertemente al movimiento universitario, pues la contrarreforma de la dictadura también desmanteló las conquistas alcanzadas en casi medio siglo de luchas e impuso un silencio que comenzó a resquebrajarse recién a mediados de la década de los ochenta, con la rearticulación popular a propósito de la profunda crisis económica y el descontento social con la dictadura, que derivó en la salida de Pinochet del poder ejecutivo en los últimos años de dicha década (Muñoz Tamayo, 2011).

El retorno a la democracia se realizó sobre las bases impuestas por la dictadura. De este modo, se mantuvo la Constitución de 1980, legitimando el orden neoliberal y la democracia protegida. La transición, durante al menos una década, estuvo marcada por las condiciones que la dictadura impuso sobre los nuevos administradores del poder político, en las que la deliberación social y la inclusión de los intereses de las clases subalternas fue negada, con el fin de resguardar la gobernabilidad y evitar los conflictos entre quienes componían la elite gobernante (Ruiz, 2015). Este cierre de las instituciones para las izquierdas fue acompañado de políticas de represión sobre quienes habían luchado activamente contra la dictadura y generaron las condiciones para su término (Goicovic, 2006).

El contexto de despolitización forzada de la población y de evidente poder e impunidad de la derecha militar generó un debilitamiento de la capacidad de intervención en la política de los movimientos sociales. En este contexto, diversas organizaciones y culturas de izquierdas se vuelcan a sus espacios más inmediatos, comenzando un proceso de regeneración del tejido social y de microluchas, que devino en una reconfiguración de las prácticas al interior del movimiento (Muñoz Tamayo, 2011). En este sentido, podemos ver cómo, con el siglo XXI, comenzó un proceso de maduración de las nuevas formas de organización al interior del movimiento estudiantil, iniciando ciclos de protestas que buscaban develar las precarias condiciones en las que funcionaba la educación pública e instalar la problemática de la desigualdad educativa y la complacencia con la que gobernaba la Concerta- 
ción, profundizando el modelo político, económico y social heredado de la dictadura (Donoso, 2005).

Las movilizaciones estudiantiles de 2006 y 2011 remecieron la sociedad chilena, instalando una crítica profunda al modelo de mercado y a la falta de representación de los intereses mayoritarios en las instituciones de representación del Estado, a contrapartida del discurso oficial que durante años habló de la sanidad de la democracia chilena y del éxito de la economía social de mercado (Fleet, 2011). Este escenario posibilitó la emergencia de nuevas fuerzas políticas, que toman distancia de las formas tradicionales de hacer y entender la política, construyendo un nuevo sentido común desde los y las excluidas por el sistema (Alvarado et al., 2019).

En este contexto de agitación social y de deslegitimación de las instituciones del Estado neoliberal chileno, resulta importante analizar no solo el desarrollo histórico del movimiento estudiantil, sino también cómo, al alero del mismo, han nacido y se han desarrollado nuevas organizaciones políticas, las que constituyen lo que Emir Sader (2018) ha calificado como la "nueva izquierda chilena", en términos de que cuestionan las lógicas de funcionamiento de las instituciones y el ordenamiento del sistema educativo, y que en los últimos años se han posicionado como alternativas políticas en el plano político-institucional, cuestionando públicamente el modelo neoliberal y abriéndose paso entre la elite política chilena mediante elecciones populares, reivindicando programas desarrollados por distintos movimientos sociales de la última década.

\section{Definiciones conceptuales}

Los conceptos centrales que se utilizaron en el presente artículo son "izquierdas emergentes" y "movimientos sociales", todo lo anterior contextualizado en la posdictadura chilena como espacio histórico, siendo el movimiento estudiantil el actor colectivo investigado. 
En primer lugar, se habla de "nuevas izquierdas" para diferenciarlas de aquellas que protagonizaron el siglo $X X$ y que vivieron sus procesos de crisis, ya por su aniquilamiento por parte de la policía secreta del Estado o por su conversión neoliberal para acomodarse en la administración del Estado e iniciar una nueva fase en el desarrollo del capital en nuestro territorio (Goicovic, 2006).

Cuando nos referimos al concepto de "nuevas izquierdas" hablamos de las organizaciones que nacen al alero de los movimientos sociales de fines del siglo XX y comienzos del XXI, que cuentan con una perspectiva transformadora de la realidad, que comprende los conflictos sociales a partir de las contradicciones entre grupos dominantes y dominados; de este modo, nutre la compresión de la contradicción capital-trabajo con los conflictos asociados a la raza, el género, el indigenismo y el medio ambiente, entre otros, para posicionarse contra el capitalismo y orientarse hacia la construcción de una sociedad socialista, sin explotadores, en la que prime la libertad, la igualdad, la solidaridad y se socialicen los medios de producción (Gómez Leyton, 2010; Harnecker, 2014). Si bien esta definición entrega elementos sustanciales que componen tanto los proyectos colectivos como las propias identidades de quienes se aglutinan en estos espacios colectivos, la tradición de las izquierdas latinoamericanas nos muestra, también, ciertas características históricas, como el internacionalismo, la solidaridad latinoamericana, el compromiso con la democracia política y económica, y la lucha por las independencias como contrapartida al imperialismo colonialista de las potencias mundiales. De igual forma, hubo falencias en común, como la falta de comprensión de las particularidades y diversidades de los territorios y los sectores sociales en los que se insertaban, lo que condujo a invisibilizar - incluso a perseguir - a las disidencias, a fin de homogenizar la lucha social bajo la noción de "clase", supeditando a las diversas expresiones de la dominación y suprimiendo el carácter político de los espacios cotidianos, relegando el poder social/popular como eje articulador de su política y 
supeditando al Estado su capacidad transformadora (Gallardo, 1993; Zibechi, 2008).

De este modo, vemos cómo el devenir histórico de las izquierdas nos muestra un periodo de crisis con el fin del siglo XX y la caída de los socialismos realmente existentes. A partir de aquello se señala que, desde la década de los noventa, surgieron nuevos actores y organizaciones políticas, cuyo horizonte emancipatorio se construyó al alero de los movimientos de resistencia al neoliberalismo. Podemos apreciar entonces cómo el concepto de "izquierdas emergentes" está en construcción por la propia acción histórica de los grupos que en ella se posicionan. Sin embargo, al buscar elementos en común entre estas diversas organizaciones y movimientos de izquierda a lo largo de América Latina, nos encontramos que, en un contexto generalizado de ruptura de consenso neoliberal, los grupos de resistencias comenzaron una progresiva articulación a partir de las luchas desarrolladas por los propios movimientos sociales que, alejados de la política institucionalizada, han construido formas de organización descentralizadas, propuestas políticas de recuperación de bienes comunes y derechos conculcados, y además una nueva relación entre el Estado y la sociedad civil, reconfigurando las formas de comprender y practicar la política (Chávez et al., 2008; Svampa, 2010).

Además, se debe reconocer que las nuevas izquierdas son heterogéneas, asumiendo formas de insurrección indígena/popular y desarrollo autónomo, hasta los denominados "gobiernos progresistas" y su apuesta estatal (Zibechi, 2010). Este es el contexto político-cultural de las nuevas izquierdas estudiadas en el presente artículo, las que se han posicionado, tensionando la relación entre política institucional y política no institucional, transitando hacia la construcción de organizaciones dispuestas a la disputa institucional. En este sentido, Chávez, et al. (2008) sostienen que existen distintas corrientes de las izquierdas emergentes de América Latina, una de ellas es la estrategia reformista respecto del Estado y el sistema capitalista, que denominaron como 
"reformismo no reformista", para diferenciarlo de las estrategias políticas de las viejas izquierdas, hoy neoliberales, y porque tienen un sentido contrahegemónico en tanto promueven reformas que destraben los anclajes antidemocráticos, para permitir un proceso de transformación continua y sostenida de las relaciones de poder social y político entre los grupos dominantes y dominados. Este tipo de reformas tiene como característica combinar intervención estatal con redistribución del ingreso y políticas públicas de planificación participativa que impulsen prácticas de poder social y profundicen la impugnación del modelo de democracia restringida (Chávez et al., 2008; Harnecker, 2014).

A partir de la revisión de las principales corrientes teóricas de los movimientos sociales, este estudio asume una perspectiva crítica y latinoamericana, en la que se destacan las nociones de "conflicto estructural" y de "praxis" emanadas de la tradición marxiana, aun cuando nos distanciamos de la centralidad asignada al conflicto capital-trabajo y, con ello, de su jerarquización de las luchas sociales que, en el caso de las disputas en el campo educativo protagonizadas por el movimiento estudiantil, las relegó - durante gran parte del siglo XX—a la categoría de "fuerzas auxiliares de la revolución", consagrando una práctica política Estadocéntrica que invisibilizó las prácticas prefigurativas y de poder subalterno que emergían desde los propios movimientos (Garcés, 2012). En este sentido, consideramos de suma importancia volcarse sobre las trayectorias históricas de los movimientos para comprenderlos, pues permite reconocer sus procesos de reflujo o aparente inactividad, en los que destruyen y construyen nuevas formas de hacer movimiento, acumulando experiencias y recursos para su posterior emergencia (Melucci, 1999).

En este sentido, rescatamos los aportes realizados por los teóricos de los nuevos movimientos sociales que, a partir de la década de los setenta, centraron sus estudios en la forma en que se desarrollan los movimientos y sus acciones colectivas, reivindicando la capacidad creativa y creadora de los/as sujetos/as sociales, que no sólo reaccionan frente a una estructura social injusta, sino que tam- 
bién cuentan con la capacidad de construir y proponer sociedades otras (Salazar, 2012). Sin embargo, cuestionamos el encuadre exclusivamente cultural que autores como Alain Touraine proponen para el estudio de los nuevos movimientos sociales, pues consideramos que coarta las posibilidades de transformación sistémica que emergen de estos propios movimientos, cuestión que, desde nuestro punto de vista, no resulta útil para los fenómenos sociales configurados en nuestro continente, pues deja de lado los conflictos contra el Estado y las relaciones entre las clases dominantes y las capas populares, cuestión que no se refiere exclusivamente a representaciones simbólicas o prácticas culturales, sino que también a la dominación en su multifactorialidad, generando resistencias en las que confluyen una diversidad de conflictos y en las cuales los movimientos, como estructuras de acción colectiva, cuentan con la capacidad de construir objetivos y acciones autónomas, dando cuenta de la producción simbólica en los múltiples planos en los que opera la dominación (García Linera, 2011).

A través de distintas investigaciones pudimos dar cuenta de los procesos de rearticulación, reflujo y resistencia del movimiento estudiantil universitario desde mediados de la década de los ochenta en adelante, en los que podemos apreciar un cambio en la relación entre el movimiento y los partidos políticos, producto de las crisis que estos enfrentaron en la conducción - y contencióndel movimiento, particularmente en los primeros años de la transición (Muñoz Tamayo, 2011). Así como también la progresiva modificación de las estructuras de organización del movimiento, producto de las crisis sufridas, cuestión que permitió la emergencia de nuevas formas, las que apuntaban a la horizontalidad y el control de la asamblea sobre sus voceras y voceros (Thielemann, 2011). De este modo, comprendemos que el movimiento estudiantil se ha configurado como un actor colectivo - inserto en un proceso de reemergencia de los movimientos a escala nacional y mundial- heterogéneo, en el que se desarrollan distintas perspectivas políticas y organizaciones, las que han posicionado discursos y acciones que denuncian la precariedad de la educación 
pública y la mercantilización/privación de este derecho fundamental. Es además un movimiento altamente rotativo en cuanto a sus integrantes, dada la configuración del sistema educativo chileno, altamente privatizado y excluyente (Farfán, 2016).

En su desarrollo histórico el movimiento fue destruyendo las formas tradicionales de organización y construyó una estructura asentada en las asambleas como espacios de discusión y decisión, avanzando, de este modo, a formas horizontales que radicalizan la democracia, tanto en la estructura como en las relaciones entre sus integrantes, y en la de estos/as con sus adversarios (Valenzuela, 2014). Consideramos además fundamental su capacidad para construir representaciones simbólicas, proyectos y acciones colectivas autónomas respecto de otras estructuras y del Estado, posicionando a sus administradores como adversarios, tanto por recursos como por poderes (Alvarado et al., 2019). En este sentido, el movimiento estudiantil de la última década se ha caracterizado por su carácter reivindicativo, al exigir la restitución de un derecho como la educación, pero también transformador, pues ha instalado exigencias que cuestionan la estructura social dominante, tanto en el plano educativo/cultural como en lo político y económico (Thielemann, 2011).

\section{Precisiones metodológicas}

Para analizar la relación entre el movimiento estudiantil de Valparaíso y la emergencia de una nueva izquierda chilena recurriremos a la reconstrucción histórica y el análisis desarrollado en la tesis de pregrado "Se nos acabó la paciencia. Memoria sobre la irrupción y configuración de las izquierdas emergentes a partir de las luchas del movimiento estudiantil universitario de Valparaíso, 2006-2016" (Briceño Arévalo, 2018). La investigación siguió una metodología cualitativa y sus principales fuentes fueron tanto documentos emanados del movimiento como entrevistas semiestructuradas realizadas a ex dirigentes de federaciones universitarias que, entre 2006 y 2016, militaron en organizaciones 
de izquierdas emergentes, es decir, se excluyen tanto a organizaciones de derechas, liberales y partidos políticos tradicionales.

Delimitamos la muestra en dos de las federaciones estudiantiles que forman parte del movimiento en la Quinta Región en Chile, particularmente la Federación de Estudiantes de la Universidad de Valparaíso (FEUV) y la Federación de Estudiantes de la Pontificia Universidad Católica de Valparaíso (FEPUCV), pues estas organizaciones estudiantiles forman parte de la Confederación de Estudiantes de Chile (CONFECH) y han ocupado cargos de vocería regional en los últimos años. Junto con ello, ambas casas de estudio representan dos componentes del sistema tradicional de educación superior; por una parte, la Universidad de Valparaíso (UV), como casa de estudios estatal, regional y autónoma, tras la reforma universitaria de 1981 y el desmembramiento de la Universidad de Chile, y por otra, la Pontificia Universidad Católica de Valparaíso (PUCV), institución privada de carácter confesional-católica, que forma parte del Consejo de Rectores de Universidades Chilenas (CRUCH), institución nacida para diferenciar a las casas de estudio tradicionales de las nacidas tras la liberalización del mercado universitario a comienzos de la década de los 80. Delimitamos la muestra a estas federaciones universitarias, pues ambas han tenido, en la última década, una relativa estabilidad orgánica, lo que nos permitió dar cuenta de la vinculación de dichos procesos de rearticulación del movimiento universitario con la formación de nuevas organizaciones políticas de izquierda que han disputado la conducción de las federaciones, fundamentalmente a partir de 2007.

A partir de la reconstrucción de la historia del movimiento pudimos identificar tres momentos históricos, en función de los cuales realizamos un diseño de muestra no probabilístico, teórico o por conveniencia (Canales, 2006). Así, como primer paso para la construcción de la muestra, realizamos una revisión de las dirigencias estudiantiles en el periodo estudiado, destacando a quienes presidieron las mesas ejecutivas y la organización a la que pertenecían, dando los siguientes datos: 
Tabla 1

Presidentela de Federación, año de mandato y organización política en la que militan

\begin{tabular}{|c|c|c|}
\hline \multicolumn{3}{|c|}{ FEDERACIONES ESTUDIANTILES PUCV } \\
\hline Año mandato & Nombre de presidente/a & Militancia de dirigente/a \\
\hline 2006 & INACTIVA & INACTIVA \\
\hline 2007 & Esteban Vega & Democracia Cristiana \\
\hline 2008 & Mauricio Araneda & Democracia Cristiana \\
\hline 2009 & Carla Amtmann & Cordón de Estudiantes \\
\hline 2010 & Jorge Sharp & Cordón de Estudiantes \\
\hline 2011 & Nataly Espinoza & Cordón de Estudiantes \\
\hline 2012 & Pablo Chamorro & Unión Nacional Estudiantil \\
\hline 2013 & Sebastián Vicencio & Unión Nacional Estudiantil \\
\hline 2014-2015 & Jorge Rauld & Izquierda Autónoma \\
\hline $2015-2016$ & Jorge Rauld & Movimiento Autonomista \\
\hline \multicolumn{3}{|c|}{ FEDERACIONES ESTUDIANTILES UV } \\
\hline Año mandato & Nombre de presidente/a & Militancia de dirigente/a \\
\hline 2006 & INACTIVA & INACTIVA \\
\hline 2007 & $\begin{array}{l}\text { Daniel Zamorano (Mesa } \\
\text { interina) }\end{array}$ & Partido Comunista \\
\hline 2008 & Ximena Muñoz & Estudiantes Movilizados \\
\hline 2009 & Gustavo Pacheco & Estudiantes Movilizados \\
\hline 2010 & Cristián Andrade & Partido Comunista \\
\hline 2011 & Sebastián Farfán & Estudiantes Movilizados \\
\hline 2012 & Marjorie Cuello & Unión Nacional Estudiantil \\
\hline 2013 & Mario Domínguez & Partido Comunista \\
\hline 2014 & $\begin{array}{l}\text { Mario Domínguez (Des- } \\
\text { tituido) }\end{array}$ & Partido Comunista \\
\hline 2014 & $\begin{array}{l}\text { Sebastián Carvajal (Mesa } \\
\text { interina) }\end{array}$ & Unión Nacional Estudiantil \\
\hline 2015 & Sebastián Carvajal & Unión Nacional Estudiantil \\
\hline 2016 & Carlos Vergara & Unión Nacional Estudiantil \\
\hline
\end{tabular}

Fuente: Briceño Arévalo (2018, pp. 49-50).

A partir de esta información diseñamos la muestra, en la que el primer criterio de exclusión fue a aquellos/as dirigentes/as militantes de partidos políticos tradicionales, pues buscábamos rescatar la trayectoria histórica de las nuevas izquierdas. Del mismo 
modo, privilegiamos entrevistar a presidentes/as o secretarios/ as generales de cada mesa ejecutiva, comprendiendo que la obtención de dicho puesto federativo responde a la generación de una correlación de fuerzas favorable, tanto a nivel general entre el estudiantado como entre las fuerzas políticas postulantes a los cargos de representación estudiantil. En los casos en que lo requirió el diseño muestral, fundamentalmente para contar con una equidad en la distribución de géneros y militancias, realizamos entrevistas a dirigentes que ocuparon el cargo de vicepresidente/a o secretario/a ejecutivo/a de cada Federación. Finalmente, nuestra muestra quedó configurada de la siguiente manera:

Tabla 2

Distribución de la muestra

\begin{tabular}{|c|c|c|c|c|}
\hline $\begin{array}{l}\text { Federa- } \\
\text { ción y año }\end{array}$ & Nombre & Cargo & $\begin{array}{l}\text { Organización en } \\
\text { que militaba }\end{array}$ & $\begin{array}{l}\text { Organización en } \\
\text { que milita el } 2017\end{array}$ \\
\hline $\begin{array}{l}\text { FEUV } \\
2008\end{array}$ & $\begin{array}{l}\text { Ximena } \\
\text { Muñoz }\end{array}$ & $\begin{array}{l}\text { Secretaria } \\
\text { General }\end{array}$ & $\begin{array}{l}\text { Coordinadora } \\
\text { Estudiantes Mo- } \\
\text { vilizados }\end{array}$ & $\begin{array}{l}\text { Independiente - } \\
\text { Movimiento de } \\
\text { Unidad Docente }\end{array}$ \\
\hline $\begin{array}{l}\text { FEPUCV } \\
2009-2010\end{array}$ & $\begin{array}{l}\text { Romina } \\
\text { Maragaño }\end{array}$ & $\begin{array}{l}\text { Secretaria } \\
\text { Ejecutiva }\end{array}$ & $\begin{array}{l}\text { Coordinadora } \\
\text { Cordón de Estu- } \\
\text { diantes }\end{array}$ & $\begin{array}{l}\text { Movimiento Au- } \\
\text { tonomista }\end{array}$ \\
\hline FEUV 2011 & $\begin{array}{l}\text { Sebastián } \\
\text { Farfán }\end{array}$ & $\begin{array}{l}\text { Secretario } \\
\text { General }\end{array}$ & $\begin{array}{l}\text { Colectivo Estu- } \\
\text { diantes Movili- } \\
\text { zados }\end{array}$ & $\begin{array}{l}\text { Nueva Demo- } \\
\text { cracia }\end{array}$ \\
\hline $\begin{array}{l}\text { FEPUCV } \\
2011\end{array}$ & $\begin{array}{l}\text { Nataly Her- } \\
\text { nández }\end{array}$ & Presidenta & $\begin{array}{l}\text { Izquierda Autó- } \\
\text { noma }\end{array}$ & $\begin{array}{l}\text { Izquierda Autó- } \\
\text { noma }\end{array}$ \\
\hline $\begin{array}{l}\text { FEUV } \\
2012\end{array}$ & $\begin{array}{l}\text { Tomás } \\
\text { Garrido }\end{array}$ & $\begin{array}{l}\text { Secretario } \\
\text { Ejecutivo }\end{array}$ & $\begin{array}{l}\text { Frente de Estu- } \\
\text { diantes Liberta- } \\
\text { rios }\end{array}$ & $\begin{array}{l}\text { Izquierda Liber- } \\
\text { taria }\end{array}$ \\
\hline $\begin{array}{l}\text { FEUV } \\
2012\end{array}$ & $\begin{array}{l}\text { Marjorie } \\
\text { Cuello }\end{array}$ & $\begin{array}{l}\text { Secretaria } \\
\text { General }\end{array}$ & $\begin{array}{l}\text { Unión Nacional } \\
\text { Estudiantil }\end{array}$ & $\begin{array}{l}\text { Nueva Demo- } \\
\text { cracia }\end{array}$ \\
\hline $\begin{array}{l}\text { FEPUCV } \\
2014-2015- \\
2016 \\
\end{array}$ & Jorge Rauld & Presidente & $\begin{array}{l}\text { Izquierda Autó- } \\
\text { noma }\end{array}$ & $\begin{array}{l}\text { Movimiento Au- } \\
\text { tonomista }\end{array}$ \\
\hline $\begin{array}{l}\text { FEPUCV } \\
2014-2015\end{array}$ & Carla Peña & $\begin{array}{l}\text { Vicepresi- } \\
\text { denta }\end{array}$ & $\begin{array}{l}\text { Frente de Estu- } \\
\text { diantes Liberta- } \\
\text { rios }\end{array}$ & $\begin{array}{l}\text { Izquierda Liber- } \\
\text { taria }\end{array}$ \\
\hline $\begin{array}{l}\text { FEUV } \\
2016\end{array}$ & $\begin{array}{l}\text { Carlos } \\
\text { Vergara }\end{array}$ & $\begin{array}{l}\text { Secretario } \\
\text { General }\end{array}$ & $\begin{array}{l}\text { Unión Nacional } \\
\text { Estudiantil }\end{array}$ & $\begin{array}{l}\text { Nueva Demo- } \\
\text { cracia }\end{array}$ \\
\hline
\end{tabular}

Fuente: Briceño Arévalo (2018, p. 53). 
Si bien identificamos que, en términos de ciclos, existen tres, hemos decidido entrevistar, dentro del periodo 2011-2014, a dos dirigentes, dada la trascendencia de las movilizaciones estudiantiles de 2011 y lo fundamental para la investigación de poder contar con dichos relatos y perspectivas.

Cabe destacar que construimos esta investigación centrada en Valparaíso, como un modo de visibilizar un territorio que ha sido trascendental para el desarrollo de movimientos sociales y populares a lo largo de nuestra historia y porque, además, consideramos relevante superar el sesgo centralista que también trasciende a la producción teórica nacional, proponiendo un análisis desde los territorios y sus particularidades.

\section{Análisis}

En su desarrollo histórico el movimiento estudiantil universitario a escala nacional puede agruparse en cuatro grandes periodos desde el comienzo de la posdictadura (Thielemann, 2011). En el primero de ellos (1988-1997), el movimiento universitario estuvo sumamente debilitado, dado el poder acaparado por los cuadros políticos militantes de partidos de la Concertación en las estructuras representativas - sumamente verticales- del movimiento. Sin embargo, este periodo de reflujo se interrumpió por las movilizaciones que reaccionaron al reimpulso de las políticas privatizadoras en el campo educativo, posicionándolo en resistencia contra las reformas gubernamentales que proponían la mercantilización como solución a la crisis económica permanente de las instituciones públicas y el sistema de créditos para financiar los estudios de los y las estudiantes más pobres (Donoso, 2005). Este proceso, de lento despertar, se enfrentó a las acciones de represión, criminalización y deslegitimación del movimiento por parte de los administradores del Estado, cuestión que marcó tanto el primer como el segundo periodo (1998-2005) que inauguramos - simbólicamente - con el asesinato de Daniel Menco durante las movilizaciones contra la Ley Marco, y concluimos con la 
derrota y deslegitimación del movimiento tras las negociaciones con Ricardo Lagos y la aprobación del Crédito con Aval del Estado (CAE) (Farfán, 2016).

La investigación que posibilita el presente artículo se centra en lo que aconteció con el movimiento universitario de Valparaíso entre el 2006 y 2016 — tercer y cuarto periodo del movimiento a escala nacional—; sin embargo, para dar cuenta de los procesos de emergencia y configuración de las nuevas izquierdas al interior del movimiento dividiremos estos periodos históricos en tres momentos.

2006-2010: Periodo de rearticulación del movimiento y emergencia de las nuevas izquierdas universitarias

A partir de la reconstrucción histórica del movimiento universitario en Valparaíso, podemos apreciar que el 2006 representa para los y las universitarias un hito, pues masificó críticas - expresadas en exigencias y discursos- que atacan el corazón del modelo de mercado en educación. Junto con ello revitalizó a sectores estudiantiles disgregados y desilusionados tras la profunda derrota que sufrió el movimiento universitario en 2005 con el ingreso de la banca para el financiamiento de los créditos, y subvirtió la formas de administrar el poder al interior del movimiento, volcando sobre las asambleas la responsabilidad de las decisiones (Briceño Arévalo, 2018).

De este modo, en Valparaíso se aprecia un proceso de aglutinamiento de los y las estudiantes de izquierdas, las primeras formas que asumieron estas agrupaciones fueron las coordinadoras, caracterizadas por reunir a distintas tradiciones de las izquierdas desde socialdemócratas disconformes con la Concertación hasta anticapitalistas en sus vertientes leninistas y libertarias. Ambas experiencias tuvieron como característica común su foco en la rearticulación de las estructuras del movimiento y la emergencia durante los procesos de movilización, instancias que las izquierdas utilizaron para instalar agendas de avanzada y formas demo- 
cráticas de toma de decisiones durante las movilizaciones, como estrategia ante la posibilidad de negociación entre partidos a espaldas del movimiento (X. Muñoz, comunicación personal, 2016).

A partir de nuestra muestra pudimos dar cuenta de las diferencias políticas entre las universidades estudiadas, pues si bien las estrategias de contención se generalizaron en los movimientos sociales a escala nacional, en la PUCV estos mecanismos fueron bastante efectivos, al menos hasta 2006, pues, por una parte, al ser una universidad de propiedad de la Iglesia cuenta con menores trabas burocráticas y de una mayor estabilidad económica al no depender exclusivamente de los fondos estatales para su funcionamiento, dada su propiedad privada y la composición de clase de sus estudiantes (Barrios, 2011). Estas características evitaban que surgieran conflictos por las precariedades, como sucede en otras instituciones de carácter regional-estatal. Por otra parte, cuenta con importantes directivos con militancias reconocidas en partidos políticos de la Concertación y aquello también ejercía influencia sobre un movimiento en reflujo y con escasa orgánica. Esto se puede apreciar en que la conducción de la federación de estudiantes estuvo, desde el plebiscito hasta 2008, en manos de la Juventud Demócrata Cristiana (JDC). Así, tras las movilizaciones contra la LGE en el 2008, resultó electa Carla Amtmann, estudiante de historia agrupada en la coordinadora Cordón de Estudiantes, siendo la primera presidenta mujer y de izquierdas en presidir la federación desde el golpe de Estado en 1973.

En tanto, la Universidad de Valparaíso (UV) vivió la emergencia de las izquierdas en medio de una profunda crisis institucional en 2007, que hizo peligrar la continuidad de esta casa de estudios, dada la fraudulenta administración del entonces rector Juan Riquelme Zucchet (Farfán, 2016). Junto con ello, debemos considerar que la UV tiene una crisis de infraestructura permanente e histórica, en la que sus 42 carreras se desarrollan en 13 sedes distribuidas por la Región de Valparaíso, lo que ha generado una cultura política donde cada espacio funciona autónomamente y por sus conflictos específicos (T. Garrido, comunicación 
personal, 2016). Esto explica que para 2007 hayan tenido desarticulada la Federación de Estudiantes, la que era integrada por los representantes de las distintas facultades, con una tendencia generalizada a la hegemonía de las izquierdas, particularmente de las Juventudes Comunistas (JJCC).

Caracterizamos este periodo como de rearticulación del movimiento universitario, pues las movilizaciones secundarias de 2006 impulsaron procesos de reorganización interna entre las y los universitarios, reconstituyéndose centros de estudiantes y federaciones estudiantiles. En este proceso se desarrollaron gran cantidad de encuentros orgánicos para dotar de estatutos y dirección política a las estructuras, y fue en esos espacios donde las convergencias programáticas permitieron el agrupamiento de las izquierdas y su diferenciación respecto de las juventudes de los partidos políticos tradicionales, reivindicando la horizontalidad como forma de organización.

Mi militancia parte como intentando levantar Centros de Estudiantes, ahí yo parto militando un poco, es como la organización social de mi Centro de Estudiantes y de mi Facultad, donde no había nada y ahí uno parte como conociendo otros cabros que son los que hoy día están militando contigo, pero no había organización, la organización la hicimos nosotros, la formulamos nosotros y aquí estamos. (R. Maragaño, comunicación personal, 2017)

De este modo, las identidades que se construyeron durante este periodo histórico entre los y las militantes de las izquierdas emergentes se arraigaron en la distancia con los partidos políticos tradicionales, reivindicando la autonomía del movimiento y una nueva concepción de la política que se vincula con sus espacios de acción inmediatos. Junto con ello, se destacó su posicionamiento crítico respecto del carácter mercantil del sistema educativo, lo que los configuró como agitadores para el desarrollo de movilizaciones, siendo el compromiso con la movilización estudiantil un núcleo identitario fundamental. 
Durante este periodo podemos ver que las izquierdas comenzaron procesos de afianzamiento programático, lo que empujó procesos de diferenciación al interior de las coordinadoras y, para el caso de la PUCV, llevó a que dos colectivos —la corriente de acción estudiantil y estudiantes autónomos- convivieran en el espacio organizativo junto a otros independientes. Mientras que en el caso de la UV las diferenciaciones llevarán al quiebre de la coordinadora de Estudiantes Movilizados (EM), la que nace durante las movilizaciones de 2007 y se constituye como colectivo tras la expulsión de las facciones disidentes a la línea marxistaleninista que hegemonizó la organización. El proceso de diferenciación al interior de las coordinadoras de estudiantes de izquierdas en Valparaíso también posibilitó el nacimiento de nuevas organizaciones y la generación de vínculos a escala regional y nacional, de este modo, al final de este periodo nos encontramos con colectivos universitarios que tienen una mayor delimitación en torno al posicionamiento ideológico y la estrategia política para el periodo, la que se caracteriza por enfocarse de manera exclusiva en la agitación política de sus propios espacios universitarios, desvinculándose de lo acontecido a escala nacional.

Esta maduración de las jóvenes organizaciones de izquierdas permitió su consolidación en las disputas de cada federación universitaria, lo que dio pie para que, ante el escenario de oposición al gobierno de derechas de Sebastián Piñera (2010-2014), se formara un tercer bloque en la disputa por la conducción de la CONFECH, en oposición a las juventudes de partidos de la Concertación y las JJCC. Este grupo se denominó por sus integrantes como sinfech, y articuló a las federaciones estudiantiles de universidades, fundamentalmente de regiones y otras precarizadas del centro del país, logrando un aumento de representación del grupo en la mesa ejecutiva de la CONFECH, pasando de un representante entre diez, a comienzos de 2010, a cuatro para finales de año. La presencia de las izquierdas en las federaciones porteñas fue vital en este proceso, dado que contaban con mejores correlaciones de fuerza a escala regional (Farfán, 2016). 
El sinfech nace del decir "hueón, aquí siempre la hegemonía la tiene la FECH y en este caso la FECH es como el PC y todo lo que nosotros hagamos tiene que ser como al margen de lo que ellos puedan hacer y las vocerías que ellos ejerzan" y ahí fue porque se divide el CONFECH y se tratan de posicionar otras vocerías que fueran por fuera de la vocería histórica de la FECH (...) como que lo desconcentra en cierta forma y le permite también darle también más consistencia a lo regional y visibilidad también, que eso ya el 2011 se materializa bien fuerte. (R. Maragaño, comunicación personal, 2017)

\section{1-2014: Periodo de conflicto, expansión y reconfiguración interna}

Si analizamos el contexto en que comenzaron a desarrollarse las movilizaciones estudiantiles de 2011, nos encontramos con un escenario social álgido, dadas las movilizaciones de distintos territorios vinculadas a problemáticas socioambientales y la disposición de distintos grupos sociales para movilizarse contra el primer gobierno de derechas de la posdictadura. En el plano educativo, nos encontramos con políticas de radicalización de privatización del sistema, a través de la disolución de la distinción entre instituciones públicas y privadas, así como distintas propuestas de reformas administrativas y financieras en un sentido mercantilizador (Centro Alerta / OPECH, 2012).

Este proceso de confluencias ante la amenaza que representaba el gobierno se expresó en el fortalecimiento del sinfech como espacio de agrupamiento de las nuevas izquierdas que operaban de manera autónoma en sus distintos espacios, pero también en la construcción de puentes de diálogo con los partidos tradicionales — liberales y socialdemócratas - fundamentalmente a través de sus altos directivos estatales, muchos de ellos decanos y rectores de instituciones de educación superior (N. Espinoza, comunicación personal, 2016). A partir de la revisión de fuentes, 
apreciamos que las demandas estudiantiles de abril de 2011 se encontraban en sintonía con las propuestas de los rectores del $\mathrm{CRUCH}$, los que exigían un posicionamiento del Ministerio de Educación y la posibilidad de participar de las instancias de construcción de la reforma (Farfán, 2016). Las estrategias asumidas por el movimiento durante esta primera fase también respondían a la correlación de fuerzas al interior de la CONFECH - como espacio de representación del movimiento a escala nacional一, en la que existía una marcada presencia de cuadros políticos de las JJCC y la Concertación en puestos dirigenciales, lo que se fue modificando durante el desarrollo de las movilizaciones, dada la creciente influencia que alcanzaron las propuestas construidas desde las universidades regionales y precarizadas, tal como se aprecia en la siguiente tabla:

Tabla 3

Composición mesa ejecutiva de la CONFECH según Federación, territorio y organización a la que se afiliaba la mesa ejecutiva.

\begin{tabular}{|l|l|l|l|l|l|}
\hline \multicolumn{3}{|c|}{ Abril 2011 } & \multicolumn{3}{c|}{ Julio 2011 } \\
\hline Federación & Región & Organización & Federación & Región & Organización \\
\hline FEUNAP & I & SINFECH & FEUNAP & I & SINFECH \\
\hline FEULS & IV & Concertación & FEUCN & IV & SINFECH \\
\hline FEUPLA & V & JJCC & FEUV & V & SINFECH \\
\hline FECH & RM & JJCC & FEUTFSM & V & SINFECH \\
\hline FEUSACH & RM & JJCC & FECH & RM & JJCC \\
\hline FEUSM & RM & JJCC & FEPUC & RM & Concertación \\
\hline FEUTEM & RM & SINFECH & FEUBB & VIII & SINFECH \\
\hline FEPUC & RM & Concertación & FEMAE & IX & SINFECH \\
\hline FEUCSC & VIII & Concertación & FEUL & X & SINFECH \\
\hline FEUL & $\mathrm{X}$ & SINFECH & & & \\
\hline
\end{tabular}

Fuente: Elaboración propia, con base en actas públicas de la CONFECH.

La distribución descentralizada de las vocerías que se aprecia a partir de julio de 2011 da cuenta de la creciente influencia de las federaciones regionales agrupadas en el sinfech, así como del poder que fueron alcanzando las bases estudiantiles, las que radi- 
calizaron las formas horizontales de organización, concurriendo en masa a las plenarias CONFECH, enviando delegados/as de asamblea e incluso revocando de los cargos federativos a quienes no cumplieron los mandatos asignados por las asambleas, tal como ocurrió con las mesas federativas de la FEULS y la FEUPLA, ambas pertenecientes a la primera mesa ejecutiva de 2011.

La discusión y la generación permanente de críticas y alternativas al modelo educativo han sido una característica de las movilizaciones estudiantiles, las que han profundizado notablemente su horizonte reivindicativo en el nuevo siglo (Reyes, 2014). En este contexto, las regiones fueron fundamentales en la profundización de las exigencias, propuestas y proyectos del movimiento. Las transformaciones que tuvo el petitorio se pueden revisar con detención en la investigación realizada por Sebastián Farfán (2016) y dan cuenta de un progreso permanente, en el cual la radicalización emerge desde las regiones e instituciones más precarizadas, las que, a través de sus propias acciones, superaron los ritmos calculados por las universidades emblemáticas y, en este proceso, Valparaíso realizó importantes aportes, como la construcción y sistematización de una propuesta de financiamiento para la gratuidad en la educación superior a través de la nacionalización de recursos naturales, redistribución del gasto del presupuesto nacional y una reforma tributaria progresiva que aumentara los impuestos a empresarios y las familias más ricas del país². Así, podemos ver cómo las consignas instaladas fueron fruto de una serie de discusiones y negociaciones al interior del propio movimiento, en las que las instituciones más precarizadas levantaron y rescataron exigencias de avanzada que fueron ganando terreno hasta construir una propuesta en rotunda oposición a las lógicas del modelo imperante. Una de esas exigencias fue la educación gratuita, la que era resistida por los sectores más

Para una revisión de la propuesta, véase https://issuu.com/caaeicouv/docs/ propuesta_financiamiento_en-cuentro_feuv 
moderados, que llamaban a bajar las movilizaciones y obtener $g a-$ nancias concretas (Farfán, 2016, p. 146).

El proceso de hegemonía de los grupos de izquierdas independientes también modificó las alianzas construidas hasta el momento. De este modo, se afianzó el bloque constituido por las juventudes de los partidos de la Concertación y las JJCC (como un preludio de la Nueva Mayoría), mientras que las izquierdas independientes continuaron trabajando en conjunto, diferenciándose entre los grupos de Izquierda Autónoma y afines, y los colectivos de izquierdas anticapitalistas, representados en las federaciones de universidades regionales y precarizadas, en las que las afiliaciones políticas pasaban por tendencias marxistas, leninistas, libertarias y autonomistas, donde se encontraba el Frente de Estudiantes Libertarios, FEL, y EM. Avendaño (2014) califica a estos sectores como pertenecientes a una corriente "radical" de izquierdas, con un marcado discurso antipartidista, así como con un posicionamiento político con demandas que superaban el conflicto educativo y cuestionaban al Estado posdictatorial en su conjunto, haciendo propia la propuesta de control comunitario de las instituciones educativas y que durante los últimos meses de movilización asumió su conducción.

La represión, criminalización y dilatación de las negociaciones por más de cinco meses por parte del gobierno de Sebastián Piñera cerró otro proceso de movilización estudiantil sin resultados para los actores educativos movilizados. Al concluir las movilizaciones, en distintos espacios se materializaron las alianzas construidas en estas, lo que permitió la inserción con fuerza del FEL en espacios de representación estudiantil. En términos generales, las movilizaciones permitieron que los grupos de izquierdas ampliaran su influencia en el campo político universitario, con el ingreso a la organización de estudiantes provenientes de los distintos espacios movilizados.

Lo que pasa es que el desgaste que se puede ver en el movimiento social o lo que fueron las manifestaciones 
no merman lo que uno aprende en las movilizaciones, entonces también esa carencia de la agitación y de cómo la pasión que se vive es encausada cuando uno ingresa a una organización social, ¿uno qué aprende? Uno aprende del compañerismo, uno quita ese individualismo, (...) estás en una organización donde tienes compañeros alrededor tuyo que están en la misma y donde las consignas no son para nosotros, "a es que yo quería educación gratuita”, sino que hoy día es cambiar esto y optar por lograr fortalecer [nuestro proyecto], por derrotar el sistema actual. (Carla Peña, comunicación personal, 2016)

De esta manera, podemos apreciar que, entre las agrupaciones de izquierdas, continuó siendo un elemento central de aglutinamiento y de construcción de sentido propio las experiencias y el compromiso con las movilizaciones sociales y las exigencias y proyectos que en ellas nacen; asimismo, apreciamos una profundización del discurso antipartidista a partir de las desconfianzas por su actuar histórico y como una crítica a las estructuras verticales de representación de la política en el Estado, por su falta de voluntad para materializar las exigencias populares expresadas en 2011. Igualmente, las posiciones de las nuevas izquierdas se masifican entre las bases estudiantiles, tornando necesaria entonces la discusión respecto de la estructura orgánica y estrategias asumidas para el periodo histórico.

Los años venideros fueron momentos de reflujo para el movimiento estudiantil, en los que el desgaste se manifestó en un descenso en el número de participantes, pues si bien se sostenían movilizaciones multitudinarias, el diálogo con el gobierno se encontraba cerrado, dadas las diferencias políticas existentes, y el movimiento volvió a su foco local vinculándose con conflictos desarrollados en los territorios o a propósito del impulso de políticas de persecución política judicial a los movimientos sociales. En Valparaíso, un conflicto que impactó fue el cierre de la Universidad del Mar en 2012, y con ello la visibilización de las 
consecuencias del desarrollo desregulado del mercado educativo. En este escenario, las izquierdas emergentes consolidaron la hegemonía alcanzada en la CONFECH durante las movilizaciones de 2011 y se enfocaron en fortalecer sus estructuras internas, así como la definición más delimitada del proyecto colectivo y las estrategias políticas para el periodo.

Las elecciones parlamentarias y presidenciales de 2013 tensionaron al movimiento en su conjunto, pues en un contexto caracterizado por la deslegitimación de los bloques gobernantes y la ruptura del consenso neoliberal promovido/impuesto por la elite, también emergieron importantes movilizaciones en las regiones más periféricas del país, manifestándose contra el alto costo de la reproducción de la vida y la progresiva devastación de los territorios. Parte de las figuras icónicas de los diversos movimientos fueron atraídas por el sistema de representación, fundamentalmente a través de los partidos políticos tradicionales; así, diversos dirigentes/as universitarios/as se vincularon a la campaña de gobierno de Michelle Bachelet, posibilitándose la apropiación de las consignas - no de los proyectos- masificadas durante las movilizaciones por parte de la Nueva Mayoría. En este contexto, las nuevas izquierdas participaron de las elecciones parlamentarias de manera disociada, obteniendo malos resultados, salvo por la victoria de Gabriel Boric como diputado, dando cuenta de las dificultades de ingreso a un sistema político sumamente blindado y elitizado como el chileno (Boccardo y Romero, 2014).

Por su parte, el movimiento secundario, a través de la ACES y otras organizaciones de izquierdas estudiantiles, posicionaron la consigna "Yo no voto por este sistema", siendo otra expresión del descontento que generó eco entre las bases estudiantiles, al tomar distancia de la concentración de los esfuerzos en posibilitar la reforma a través de los canales institucionales y llamó a la profundización del proyecto alternativo nacido del movimiento estudiantil. Consideramos que esto es significativo porque da cuenta de las distintas posiciones que subyacen en el movimien- 
to estudiantil y explica las tensiones y quiebres que sufrieron las organizaciones de nuevas izquierdas tras disponerse a disputar cargos de representación. Y si bien los resultados no fueron los esperados por ellos, el paso dado por las izquierdas emergentes fue definitivo en asumir la vía institucional como estrategia de transformación.

Durante este periodo se consagró entre las nuevas izquierdas una identidad vinculada a la de su generación, al ser su condición de juventud una primera característica en común, así como también la masificación del acceso a las universidades a través del endeudamiento y, con ello, la constitución de generaciones que ven frustradas las posibilidades de mejorar sus condiciones de vida y reproducción a través de la educación, pues el mercado desregulado pauperiza las condiciones de trabajo para las y los nuevos profesionales (Ruiz, 2015). Sumemos a esto que su participación en las movilizaciones estudiantiles de 2006 y/o 2011 generó una profunda desconfianza con los mecanismos de resolución de conflictos por parte del Estado, oponiéndose a sus administradores — caracterizados como el duopolio- y autoafirmándose como representantes generacionales del malestar (Alvarado et al., 2019).

\section{4-2016: Ampliación de bases sociales y preparación del asalto electoral}

En términos generales, este periodo es de latencia para el movimiento, en tanto los grupos dominantes lograron cooptar las consignas de los estudiantes para legitimar proyectos de ley que no modificaban la estructura ni la lógica de funcionamiento del sistema educativo. La alta aprobación y legitimidad de las figuras icónicas de la movilización estudiantil con la presidenta electa Michelle Bachelet (2014-2018) generaron un escenario de reflujo que al movimiento le costó abordar. Durante este periodo podemos apreciar un reordenamiento de las fuerzas que componen el campo político universitario en Valparaíso, pues a 
la unión entre las juventudes de la Concertación y el PC bajo el paraguas de la Nueva Mayoría se sumó el ingreso en la disputa de las derechas y sus organizaciones universitarias, que en el caso de la PUCV disputará con fuerza las elecciones a través del Movimiento Gremialista. Este contexto generó en el campo de las izquierdas la necesidad de unificar sus esfuerzos, generándose las alianzas entre los grupos de izquierdas más mayoritarios, alcanzando los mandatos de las mesas ejecutivas en la región a través de la alianza FEL, UNE e IA, cuestión que años más tarde se replicó para la emergencia del Frente Amplio.

[La PUCV] tiene un sector conservador importante tanto en la conducción de la Universidad como en el mundo estudiantil, entonces requiere una articulación de la izquierda, ahora no cualquier articulación, no una articulación ficticia como de unidad por unidad, sino que entendiendo un proyecto común que planteaba en ese momento [2014] el enfrentar la reforma, construir organización con los trabajadores, disputar la misma conducción que tenía la Universidad y que nos permitió ir en conjunto nuevamente para disputar esas discusiones. (J. Rauld, comunicación personal, 2017)

Se puede apreciar que, pese a los esfuerzos por levantar movilizaciones, estas no lograron estallar a escala nacional, ante la respuesta permanente del gobierno de institucionalizar el conflicto a partir del traslado del debate respecto a la reforma educativa a la discusión parlamentaria, donde los movimientos sociales cuentan con escasa representación. Así, las movilizaciones durante este periodo se concentraron en elementos internos de cada universidad. Este proceso de reflujo nacional y movilizaciones internas esporádicas posibilitó la construcción de distintos proyectos alternativos entre las bases estudiantiles y, con ello, se fue dotando de contenido político al proyecto alternativo del propio movimiento. Una de las muestras fue la publicación de los Principios fundamentales para una nueva educación pública, en 2015, 
documento que sintetiza las propuestas educativas de la CONFECH y que, en una visión sistémica, propone una nueva configuración y administración de la educación chilena, prohibiendo el lucro, limitando a los agentes privados, construyendo una red de educación pública de escala nacional y bajo administración de sus comunidades, y donde los conocimientos se vinculen a una estrategia nacional de desarrollo, promoviendo una educación integradora, democrática y no sexista (CONFECH, 2015).

Para 2016 podemos apreciar que, a nivel regional, las alianzas entre el FEL, Movimiento Autonomista y la UNE continuaron garantizando la hegemonía de las nuevas izquierdas en las federaciones universitarias, mientras que a nivel nacional se desarrolló un proceso de acercamiento con Revolución Democrática (RD) —que durante 2015 tomó distancia con el gobierno de la Nueva Mayoría-, agrupándose con estas organizaciones que se autodenominaron como el "bloque de conducción", asumiendo la tarea de proponer una fase de transición de la universidad neoliberal a la universidad reformada (M. Cuello, comunicación personal, 2016). Esto se vincula con el proceso de agrupación política que impulsan estas organizaciones, para construir una coalición que permita agrupar a fuerzas de izquierdas y liberales-progresistas en la disputa en el plano político institucional.

Para 2016 se visualiza la consolidación de las organizaciones feministas que, en el caso de la UV, las lleva a integrar la mesa interina en 2015 y a promover - junto a otras - una reestructuración descentralizada de la FEUV a través de la creación de vocalías temáticas —entre ellas la vocalía de género-, independientes de las listas de federación en las que se concentraba la disputa entre las juventudes de la Nueva Mayoría y de las nuevas izquierdas. De este modo, durante este periodo podemos apreciar como surgen nuevas organizaciones que se desmarcan de la vía institucional propuesta por esta nueva izquierda, cuestión que da cuenta del dinamismo del movimiento, pero también del distanciamiento que, inevitablemente, ocurre cuando las izquierdas emergentes alcanzan una complejidad orgánica que transforma a 
los antiguos colectivos en frentes de masas ${ }^{3}$ desde los cuales articularse. En este sentido, podemos apreciar que, durante este periodo, se consagra una estrategia reformista, centrando la acción política en la inserción institucional a partir de las elecciones.

Este proceso de agrupamiento de las izquierdas se realiza bajo un posicionamiento antineoliberal que recoge las exigencias levantadas por distintos movimientos sociales durante la posdictadura, y propone gestionarlas al interior del aparato legislativo. En cuanto a las figuras de auto representación que estas nuevas izquierdas han creado, estas se vinculan a la idea de izquierdas ciudadanas, revindicando el carácter movimental de su nacimiento y su disposición por impugnar el funcionamiento, los significados y contenidos del sistema político postransicional (Alvarado et al., 2019).

El desafío político más transversal es cómo surge una alternativa que pueda redefinir, redibujar a la izquierda que tradicionalmente hemos conocido, dando un carácter, no sé si ciudadano, porque está tan manoseado y descafeinado eso, pero dándole un contenido transformador a eso, porque esa discusión no se ha dado y si bien el concepto de "ciudadano" todos lo ocupan, porque es un sello como interesante, pero el contenido de eso, el contenido transformador, el contenido revolucionario, de hombre nuevo, hay que dárselo. (R. Maragaño, comunicación personal, 2017)

En octubre de 2016 resulta electo el ex dirigente estudiantil y militante autonomista Jorge Sharp como alcalde de Valparaíso, cuestión que marca la primera victoria de estas nuevas izquier-

En 2015 el FEL se transforma en el frente estudiantil del movimiento Izquierda Libertaria, mientras que la UNE pasa a ser, en 2017, el brazo estudiantil del partido Nueva Democracia. Por su parte, IA mantuvo su centralidad en lo estudiantil y la disidencia expulsada conformó el Movimiento Autonomista, desde donde ganó la Alcaldía de Valparaíso y se conformó como un movimiento político con multiplicidad de bases sociales. 
das, lo que las llevará a constituir, en enero de 2017, el Frente Amplio como alternativa política para las elecciones presidenciales y parlamentarias del mismo año. Este hito, creemos, representó el comienzo de un nuevo ciclo para las nuevas izquierdas, las que para este momento contaban con estructuras nacionales de representación que superaban el plano estudiantil, y se concentraban en la disputa institucional al transformarse durante 2018 y 2019 en partidos políticos legales.

\section{Conclusiones}

A partir de lo señalado es que podemos apreciar que en el periodo 2006-2016 se generó un desarrollo cualitativo del movimiento estudiantil universitario de Valparaíso, tanto por las coyunturas de movilización a escala nacional y local como por los grados de posicionamiento en el proyecto en construcción. Podemos apreciar cómo la negativa permanente por parte del Estado de dar solución a las problemáticas y de acoger las exigencias y propuestas del movimiento estudiantil han permitido la profundización del proyecto reivindicativo del mismo, consagrándose en los últimos años una propuesta que no solo apela a la falta de financiamiento de la educación pública, sino que consagra la necesidad de restituir el derecho a la educación pública a través de un sistema integrado, democrático, estatal, gratuito y no sexista.

Podemos observar que hasta el año 2013 no existía una mayor diferenciación entre los proyectos políticos de las nuevas izquierdas y el del movimiento estudiantil, pues el campo de acción de sus activistas estaba circunscrito a sus propias universidades; sin embargo, ya para el tercer periodo (2014-2016) comenzaron a generarse mayores grados de diferenciación, básicamente por la vinculación de estas nuevas izquierdas estudiantiles con organizaciones extraestudiantiles y su estrategia de inserción institucional, todo en gran medida posibilitado por la visibilización y referenciación pública que le dieron las vocerías $\mathrm{CONFECH}$ durante las movilizaciones. 
Nos parece relevante destacar, sobre la base de la heterogeneidad y la alta rotación que caracteriza al movimiento, que las izquierdas emergentes, al constituirse como bloque hegemónico, no totalizan el campo político universitario, ni siquiera el de las izquierdas universitarias, pues de manera permanente siguen emergiendo nuevas organizaciones que les disputan posiciones desde el mismo sector; en este sentido, parece relevante considerar el último periodo estudiado (2014-2016), pues puede verse como un preludio de lo acontecido en los últimos años con el movimiento universitario y las organizaciones feministas: si los movimientos de regiones y las izquierdas emergentes fueron capaces en el pasado de rebasar las estructuras de representación estudiantil, hoy esas mismas organizaciones han sido rebasadas por otras nuevas, esta vez feministas autónomas, que se desmarcan de las izquierdas apostando por una radicalización de la democracia interna del movimiento. De este modo, subvierten las lógicas de organización y demanda, en tanto no sólo exigen al Estado, sino que impulsan procesos de cuestionamientos y reconfiguraciones internas con la finalidad de atacar los patrones patriarcales que aun operan en los grupos subalternos.

En términos de características, podemos ver que la estrecha vinculación del movimiento universitario porteño con las organizaciones de nueva izquierda generó una relación dialéctica en la cual, al ampliarse las bases sociales y el radio de acción de esta nueva izquierda, se constituyeron identidades que provenían de la ciudadanía movilizada y decepcionada con el sistema político chileno. Esto ha dado paso a una progresiva unificación de movimientos y a la construcción de un programa político anticapitalista que se articula desde lo antineoliberal con el fin de ampliar las bases de apoyo. Asimismo, asumen como estructura orgánica el centralismo-democrático, ampliando la diferenciación entre dirigentes y dirigidos y la división de los trabajos al interior de cada orgánica. Si bien la inserción de estas agrupaciones en el Estado los ha conducido a asumir formas cada vez más verticales, podemos ver que mantienen mecanismos de revocabilidad vigentes, 
con el fin de garantizar un control democrático de los asuntos tratados por los dirigentes (Harnecker, 2014).

Lo anterior, se vincula con el tercer elemento a destacar de las izquierdas emergentes, que se refiere a las estrategias que han asumido en el último periodo, en el que se han abocado a la disputa de espacios de poder con las clases dominantes, entrando de lleno en las elecciones de cargos institucionales. Ello ha tensionado su relación con los movimientos de los que forman parte, visualizándose el peligro de la instrumentalización y conducción para sus fines particulares, dada sus ansias de emergencia.

Dicho lo anterior, nos preguntamos: ¿cuál es la importancia del movimiento universitario y sus luchas para el surgimiento de estas nuevas izquierdas?

En primer lugar, consideramos que el movimiento estudiantil constituye un campo fértil en el que las nuevas izquierdas han logrado desarrollarse y en el cual se han refugiado en los periodos de mayor debilidad, logrando rearticularse y robustecerse a partir de las luchas del movimiento universitario. Para el caso de Valparaíso, podemos analizar que desde 2006 ha existido un exponencial desarrollo de las nuevas izquierdas, las que hasta el año 2016 se mantenían en la conducción de sus respectivas federaciones universitarias. En segundo término, el movimiento estudiantil ha sido una trinchera de lucha desde la que han logrado, mediante movilizaciones masivas y creativas, convocar a amplios sectores de la sociedad y romper con el sentido común neoliberal, al cuestionar los principios sobre los que se erige el modelo y reivindicar el bien común como elemento sustancial para la legitimidad de los actores en lucha (Svampa, 2010). En este sentido, la significación histórica del movimiento estudiantil es que este logró romper la hegemonía de dominación instalada desde los noventa, cuestionando los pilares del neoliberalismo y su funcionamiento en el campo educativo, cuestión que ha sido capitalizada por las nuevas izquierdas, posicionadas como la representación institucional de dicha disputa de sentidos (Fleet, 2011). 
En tercer lugar, la importancia del movimiento estudiantil para las izquierdas emergentes también radica en la relación dialéctica que han construido, en tanto éstas forman parte importante de las fuerzas políticas que disputan la conducción del movimiento y al mismo tiempo se ven fuertemente influenciadas por las luchas y tensiones generadas en su seno. En este sentido, no es posible comprender las evoluciones de las nuevas izquierdas sin considerar el devenir histórico del movimiento estudiantil en la última década $\mathrm{y}$, pese a que las estas izquierdas han evolucionado desde agrupaciones exclusivamente universitarias a proyectos políticos de escala mayor, su principal campo de acción y visibilidad continúa estando en el terreno político estudiantil universitario, lo que genera una permanente renovación de las militancias, relecturas de sus desafíos y propuestas. En definitiva, no son organizaciones estáticas, sino que en permanente renovación e inevitables transformaciones, dado que su base social aún depende, en gran medida, de los espacios universitarios, pues tal como señalan los entrevistados, el movimiento estudiantil ha sido (y es) su cuna.

\section{Referencias}

Alvarado, E., Rivera, P. y Morales, R. (2019). Radicalizar la democracia desde los movimientos sociales. Los casos comparados de Podemos en España y del Frente Amplio en Chile. Izquierdas, (48), 87-105.

Avendaño, O. (2014). Fracturas y representación política en el movimiento estudiantil. Chile 2011. Última Década, (41), 41-68.

Barrios, M. (2011). Deserción y financiamiento en las universidades chilenas (Tesis de Magister). Pontificia Universidad Católica de Chile, Santiago de Chile.

Boccardo, G. y Romero, N. (2014). Entre la restauración y el arranque de una transformación de la política ¿Se inicia un nuevo ciclo político en Chile? Cuaderno de coyuntura, (3), 4-17.

Briceño, A. (2018). Se nos acabó la paciencia. Memoria sobre la irrupción y configuración de las izquierdas emergentes a partir de las luchas del movimiento estudiantil universitario de Valparaíso (Tesis de pregrado). Universidad de Valparaíso, Valparaíso. 
Canales, M. (2006). Metodologías de la investigación social. Santiago de Chile: LOM.

Centro Alerta / OPECH. (2012). Contextualización 2011. En J. González, R. Sánchez y M. Sobarzo comp. 2011: Aportes para interpretar una década de lucha por autoeducación (pp. 17-22). Santiago de Chile: Editorial Quimantú.

Chávez, D., Rodríguez Garavito, C. y Barrett, P. (2008). La nueva izquierda en América Latina. Madrid: Catarata.

CONFECH. (2015). Principios fundamentales para nueva educación pública. Recuperado de http://www.uchile.cl/documentos/principios-para-una-nueva-educacion-confech_113390_8_2208. pdf

De Sousa Santos, B. (2008). La universidad en el siglo XXI: Para una reforma democrática y emancipadora de la universidad. Caracas: Centro Internacional Mirada.

Donoso, S. (2005). Reforma y politica educacional en Chile 1990-2004: El neoliberalismo en crisis. Estudios pedagógicos, 113-135.

Farfán, S. (2016). "Queremos el mundo": Continuidad y cambio en las demandas de la CONFECH durante la primavera de Chile el 2011. (Tesis de Pregrado). Universidad de Valparaíso, Valparaíso.

Fleet, N. (2011). Movimiento estudiantil y transformaciones sociales en Chile: una perspectiva sociológica. Polis, Revista de la Universidad Bolivariana, 10(30), 99-116.

Gallardo, H. (1993). Elementos para una discusión sobre la izquierda política en América Latina. Revista Pasos, (50).

Garcés, M. (2012). El despertar de la sociedad. Los movimientos sociales en América Latina y Chile. Santiago de Chile: LOM.

García Linera, Á. (2011). La potencia plebeya. La Habana: Fondo Editorial Casa de las Américas.

Giroux, H. (2011). Más allá del fraude de la universidad corporativa: La educación superior al servicio de la democracia. Recuperado de http:/ / www.rebelion.org/noticia.php?id=120837

Goicovic, I. (2006). La refundación del capitalismo y la transición democrática en Chile (1973-2004). HAOL, 10, 7-16.

Gómez Leyton, J. C. (2010). Política, democracia y ciudadanía en una sociedad neoliberal (Chile: 1990-2010). Santiago de Chile: Editorial ARCIS. 
Harnecker, M. (2014). Un mundo a construir: Nuevos caminos. Santiago de Chile: LOM.

Melucci, A. (1999). Acción colectiva, vida cotidiana y democracia. México D.F.: Colegio de Profesores de México.

Muñoz Tamayo, V. (2011). Generaciones: Juventud universitaria e izquierdas politicas en Chile y México. Santiago de Chile: LOM.

OPECH. (2009). De actores secundarios a estudiantes protagonistas. Santiago de Chile: $\mathrm{OPECH}$.

Reyes, L. (2014). La escuela en nuestras manos. Santiago de Chile: Editorial Quimantú.

Ruiz, C. (2015). De nuevo la sociedad. Santiago de Chile: LOM.

Salazar, G. (2012). Movimientos sociales en Chile. Santiago de Chile: Uqbar Editores.

Svampa, M. (2010). Movimientos sociales, matrices socio-políticas y nuevos escenarios en América Latina. OneWorld Perspective, Working papers, 1, 1-29.

Thielemann, L. (2011). Para una periodificación del movimiento estudiantil de la transición (1990-2011). Revista Pretérico Imperfecto, 1-12.

Tijoux, M. E. (2011). Teoría y movimientos sociales. En MPL y Corporación Poblar (Eds.), Latinoamericamente (pp. 33-44). Santiago de Chile: Quimantú.

Valenzuela, K. (2014). La primavera de Chile y sus matices: análisis del Movimiento Estudiantil y sus tensiones entre la política "desde abajo" y la apuesta institucional. (Avance de Tesis de Doctorado). Universidad de Nottingham, Nottingham, Inglaterra.

Zibechi, R. (2008). Autonomías y emancipaciones, América Latina en movimiento. Santiago de Chile: Quimantú.

Zibechi, R. (2010). Progresismo. La domesticación de los conflictos sociales. Santiago de Chile: Editorial Quimantú. 